\title{
Como a Política Nacional de Controle do Tabaco Pode Ajudar no Enfrentamento da Pandemia da Covid-19?
}

doi: https://doi.org/10.32635/2176-9745.RBC.2020v66nTemaAtual.1023

How can the National Tobacco Control Policy Help to Tackle the COVID-19 Pandemic?
¿Como la Política Nacional de Control del Tabaco Podría Ayudar a Enfrentar la Pandemia de Covid-19?

Tânia Maria Cavalcante'; Felipe Lacerda Mendes²; Cristina de Abreu Perez ${ }^{3}$; Alexandre Octavio Ribeiro de Carvalho ${ }^{4}$; Ana Paula Leal Teixeiras; João Ricardo Rodrigues Viegas ${ }^{6}$

\section{INTRODUÇÃO}

A pandemia do novo coronavírus (2019-nCoV), responsável pela síndrome respiratória aguda grave do coronavírus 2 (severe acute respiratory syndrome coronavirus 2 - Sars-CoV-2), representa uma das maiores crises globais dos últimos tempos, frente à sua rápida transmissibilidade, à sua gravidade para os grupos mais vulneráveis, e, sobretudo, frente à atual inexistência de vacina e tratamento de eficácia comprovada.

Entre os grupos de risco, identificados como mais vulneráveis para as complicaçôes de saúde decorrentes da doença pelo coronavírus 2019 (coronavirus disease 2019 Covid-19), estão os fumantes. As evidências confirmam que o tabagismo está associado a uma maior possibilidade de progressões negativas e resultados adversos na infecção pelo Sars-CoV-2 $2^{1-3}$. Além disso, o repetido contato dos dedos com os lábios, durante o ato de fumar, pode aumentar a possibilidade da transmissão do vírus, caso as mãos ou o próprio cigarro estejam infectados ${ }^{4,5}$.

Enquanto a pandemia da Covid-19 é recente e rapidamente mobilizou recursos e vontade política para seu enfrentamento em todo o planeta, o tabagismo é uma pandemia antiga, de evolução crônica e arrastada, apesar de todo o conhecimento sobre seus riscos e as melhores práticas para revertê-la.

Hoje, o tabagismo responde por oito milhóes de mortes anuais ${ }^{6}$, das quais, 157 mil ocorrem no Brasil ${ }^{7}$. As doenças causadas pelo tabagismo geram, no país, um custo público anual de $\mathrm{R} \$ 57$ bilhốes com tratamento e pensôes ${ }^{7}$. No entanto, a importância das medidas de controle do tabaco, como estratégia de enfrentamento da disseminação e das complicaçôes da Covid-19, ainda tem recebido pouca atenção.

No final dos anos 1990, o tabagismo foi reconhecido como uma doença pediátrica, pois cerca de $90 \%$ dos fumantes se tornam dependentes da nicotina ainda na adolescência, como efeito das açôes de marketing dos produtos de tabaco promovidas pelas fabricantes de cigarros $^{8}$. No ano 2000, a Organização Mundial da Saúde (OMS) chegou a declarar que a propaganda e a promoção dos produtos de tabaco tornaram o tabagismo uma doença transmissível ${ }^{9}$. A esse conceito somou-se o de que a indústria do tabaco atua como um vetor da pandemia de tabagismo, incluindo-se tanto o marketing dirigido aos jovens, como suas práticas de interferência para minar políticas públicas ${ }^{10-13}$. A seguir, os países consolidaram o entendimento de que era preciso lidar com os determinantes transnacionais relacionados às práticas de mercado, que contribuíam para a expansáo global desse consumo.

Frente a esse complexo cenário, em 2003 foi aprovada a Convenção-Quadro da OMS para Controle do Tabaco (CQCT/OMS) - primeiro tratado internacional de saúde pública. A Convenção está em vigor há 15 anos e seu mais recente relatório de progresso mostrou, pela primeira vez, uma redução relativa de $30 \%$ na prevalência global de fumantes ${ }^{14}$. No entanto, frente à grave morbimortalidade do tabagismo, essa resposta pode ser considerada lenta, e uma das causas apontadas pelos países é a interferência da indústria do tabaco ${ }^{15}$.

'Médica. Doutora pelo Programa de Pós-Graduação Stricto Sensu em Oncologia do Instituto Nacional de Câncer José Alencar Gomes da Silva (INCA). Rio de Janeiro (RJ), Brasil. Orcid iD: https://orcid.org/0000-0003-0866-4672

${ }^{2}$ Bacharel em Direito. Mestrando em Saúde Pública pela Escola Nacional de Saúde Pública Sérgio Arouca. Fundação Oswaldo Cruz (Ensp/Fiocruz). INCA. Rio de Janeiro (RJ), Brasil. Orcid iD: https://orcid.org/0000-0003-1071-1597

${ }^{3}$ Psicóloga. Doutoranda em Saúde Pública pela Ensp/Fiocruz. INCA. Rio de Janeiro (RJ), Brasil. Orcid iD: https://orcid.org/0000-0003-1071-1597

${ }^{4}$ Historiador. Mestre em História Contemporânea pela Fundação Getúlio Vargas. INCA. Rio de Janeiro (RJ), Brasil. Orcid iD: https://orcid.org/0000-0001-5287-8884

${ }^{5}$ Administradora. Mestre em Saúde Pública pela Ensp/Fiocruz. INCA. Rio de Janeiro (RJ), Brasil. Orcid iD: https://orcid.org/0000-0002-7400-8852

${ }^{6}$ Bacharel em Relações Internacionais. Doutor em Ciência Política pelo Instituto Universitário de Pesquisas do Rio de Janeiro (IUPERJ)/Candido Mendes. INCA. Rio de Janeiro (RJ), Brasil. Orcid iD: https://orcid.org/0000-0002-0571-2969

Endereço para correspondência: Felipe Lacerda Mendes. Rua Guajaratuba, 71, apto. 102 -Tijuca. Rio de Janeiro (RJ), Brasil. CEP 20511-300. E-mail: felmendes@yahoo.com.br 


\section{DESENVOLVIMENTO}

\section{A POLÍTICA NACIONAL DE CONTROLE DO TABACO}

No Brasil, em 2005, a implementação da CQCT/ OMS tornou-se a Política Nacional de Controle do Tabaco, uma política de Estado. Entre 2006 e 2019, a prevalência de fumantes no Brasil caiu de $15,7 \%$ para $9,8 \%{ }^{16}$, o que contribuiu de forma significativa para reduzir a mortalidade por doenças cardiovasculares, pulmonares crônicas e câncer de pulmão ${ }^{17}$. A realização de açôes educativas e o tratamento para cessaçáo de fumar no Sistema Único de Saúde (SUS), juntamente com a aprovação de leis relacionadas à prevenção do tabagismo, foram fundamentais para esses resultados. Entre as Leis, destacam-se: a proibição de fumar em recintos coletivos; o aumento de impostos federais e a adoçáo de política de preços mínimos para cigarros; a proibição de propaganda, promoçáo e patrocínio de produtos de tabaco; a inserção de advertências sanitárias com fotos nas embalagens; a regulamentação dos conteúdos e emissões dos produtos. As medidas adotadas entre 1989 e 2010 evitaram 420 mil mortes pelo tabagismo ${ }^{18}$.

Se por um lado os resultados e efeitos positivos da Política Nacional de Controle do Tabaco são comemorados nacional e internacionalmente; por outro, não se pode deixar de reconhecer que os avanços ocorreram a passos muito lentos, em funçáo do confronto de forças entre as empresas de tabaco e a vontade política dos tomadores de decisão ${ }^{19}$. Por exemplo, os ajustes na legislação federal para proibir o ato de fumar em recintos coletivos, protegendo a populaçáo dos riscos do tabagismo passivo, levaram sete anos para serem aprovados no Congresso Nacional, em decorrência do lobby da indústria do tabaco ${ }^{20}$.

Já a medida da Agência Nacional de Vigilância Sanitária, que proíbe o uso de aditivos saborizantes nos cigarros, vem sendo postergada desde 2012 por entidades que ajuizaram açóes de inconstitucionalidade no Supremo Tribunal Federal ${ }^{21}$.

Por sua vez, a aprovaçáo do Projeto de Lei do Senado n. ${ }^{\circ}$ 769/2015 levou quatro anos para seguir à Câmara dos Deputados, em função de subsequentes manobras de parlamentares da bancada do fumo ${ }^{22}$. Esse projeto visa a proibir o uso de aditivos que dáo sabores aos cigarros e a instituir a padronizaçáo das embalagens de produtos de tabaco, para neutralizar a utilização de maços coloridos e cheirosos como ferramenta para atrair crianças e adolescentes, conforme descrevem as empresas em documentos internos ${ }^{23-25}$.

Como consequência desses retardos, apesar da queda relevante na prevalência de fumantes no país, o atual patamar do tabagismo ainda se traduz em um elevado número absoluto de fumantes: 20 milhóes, quase duas vezes a população de Portugal e sete vezes a população do Uruguai. São indivíduos que, em razão da dependência da nicotina, se expóem diariamente a milhares de substâncias tóxicas da fumaça de tabaco, estáo sob o risco de desenvolverem doenças graves, como as cardiovasculares, as pulmonares obstrutivas crônicas e câncer, e atualmente sob risco de infeção pela Covid-19. Importante destacar que o elevado custo do tabagismo para o Brasil tenderá a aumentar em função de uma maior pressão por assistência hospitalar e insumos para o tratamento da Covid-19 entre fumantes.

\section{CONCLUSÃO}

É fundamental que as estratégias nacionais para enfrentamento da Covid-19 incluam medidas capazes de reduzir ainda mais o número de fumantes no Brasil. Isso pode ser feito com investimentos no aumento da cobertura do tratamento para cessação de fumar, oferecido pelo SUS, e com medidas eficazes para prevenir a iniciação de jovens no tabagismo.

Nesse sentido, a Agenda Legislativa Federal deve incluir a apreciação e a aprovação urgente de projetos de Lei que têm o potencial de contribuir para o combate à Covid-19, incluindo-se o aumento da tributaçáo sobre cigarros, que reduz sua acessibilidade, e a vinculação de parte da arrecadação dos tributos para financiar as açóes de saúde.

Sobretudo, a vontade política dos Poderes Legislativo e Executivo são fundamentais para vencer o lobby e a interferência da indústria do tabaco. $\mathrm{O}$ persistente retardo na implementação de políticas para reduzir o tabagismo no país certamente amplia seu custo econômico e social. Somem-se, a estes, os custos do impacto do tabagismo sobre a pandemia da Covid-19, ambos ainda não estimados.

\section{CONTRIBUIÇÕES}

Tânia Maria Cavalcante contribuiu substancialmente na concepção do estudo; na obtenção, na análise e interpretação dos dados; na redação e revisão crítica. Felipe Lacerda Mendes e Cristina de Abreu Perez contribuíram na obtenção, na análise e interpretação dos dados; na redação e revisão crítica. Alexandre Octavio Ribeiro de Carvalho, Ana Paula Leal Teixeira e Joáo Ricardo Rodrigues Viegas contribuíram na revisáo crítica. Todos os autores aprovaram a versão final a ser publicada.

\section{DECLARAÇÃO DE CONFLITO DE INTERESSES}

Nada a declarar.

\section{FONTES DE FINANCIAMENTO}

Não há. 


\section{REFERÊNCIAS}

1. Vardavas CI, Nikitara K. COVID-19 and smoking: a systematic review of the evidence. Tob Induc Dis. 2020 March;18:20. doi: https://doi.org/10.18332/tid/119324

2. Szklo AS. Associação entre fumar e progressão para complicaçóes respiratórias graves em pacientes com covid-19. Rev Bras Cancerol. 2020;66(2):e03974. doi: https://doi.org/10.32635/2176-9745. RBC.2020v66n2.974

3. Patanavanich R, Glantz SA. Smoking is Associated with COVID-19Progression: a meta-analysis. MedRxiv. 2020 Apr 16. doi: https://doi.org/10.1101/2020.04.13.20063669

4. World Health Organization [Internet]. Geneva: WHO; c2020. Q\&A on smoking and COVID-19: are smokers and tobacco users at higher risk of COVID-19 infection?; 2020 Mar 24 [cited 2020 abr 22]. Available from: https:// www.who.int/news-room/q-a-detail/q-a-on-smokingand-covid-19

5. Framework Convention on Tobacco Control Secretariat [Internet]. Beirute: FCTC; 2019. Increased risk of COVID-19 infection amongst smokers and amongst waterpipe users; [2020] [cited 2020 Apr 22]. Available from: https://untobaccocontrol.org/kh/waterpipes/ covid-19/

6. World Health Organization. WHO report on the global tobacco epidemic, 2019: offer help to quit tobacco use [Internet]. Geneva: WHO; 2019 [cited 2020 Mar 31]. Available from: https://apps.who.int/iris/rest/ bitstreams/1239531/retrieve

7. Pinto M, Bardach A, Palacios A, et al. Carga de doença atribuível ao uso do tabaco no Brasil e potencial impacto do aumento de preços por meio de impostos [Internet]. Buenos Aires: Instituto de Efectividad Clínica y Sanitaria; 2017. (Documento técnico IECS; no. 21) [acesso 2020 abr 20]. Disponível em: https://www.iecs.org.ar/wpcontent/uploads/Reporte-completo.pdf

8. Committee on Environmental Health, Committee on Substance Abuse, Committee on Adolescence, and Committee on Native American Child Health. Policy statement - Tobacco use: a pediatric disease. American Academy of Pediatrics [Internet]. 2009 [cited 2020 Apr 30];124(5):1474-87. Available from: https://pediatrics. aappublications.org/content/pediatrics/124/5/1474. full-text.pdf

9. Pennington $\mathrm{M}$. WHO goes global against tobacco. CBS News [Internet]. 2000 May 31 [cited Apr 30]. Available from: https://www.cbsnews.com/news/who-goes-globalagainst-tobacco

10. Taylor AL, Bettcher DB. WHO Framework Convention on Tobacco Control: a global "good" for public health. Bull World Health Organ [Internet]. 2000 [cited 2020 Apr 21];78(7):920-9. Available from: https://www. scielosp.org/pdf/bwho/2000.v78n7/920-929/en
11. Samet JM. A global health perspective on the future of tobacco control. Salud Publica Mex. 2012;54(3):264-9. doi: https://doi.org/10.1590/s003636342012000300008

12. Lee S, Ling PM, Glantz SA. The vector of the tobacco epidemic: tobacco industry practices in low and middleincome countries. Cancer Causes Control. 2012;23 Suppl 1:117-29. doi: https://doi.org/10.1007/s10552012-9914-0

13. Molyneux A, Lewis S, Antoniak M, et al. Is smoking a communicable disease? Effect of exposure to ever smokers in school tutor groups on the risk of incident smoking in the first year of secondary school. Tob Control. 2002;11(3):241-5. doi: https://doi.org/10.1136/ tc. 11.3 .241

14. WHO Framework Convention on Tobacco Control. 2018 global progress report on implementation of the WHO Framework Convention on Tobacco Control [Internet]. Geneva: WHO; 2018. [cited 2020 Apr 30]. Available from: https://www.who.int/fctc/reporting/ WHO-FCTC-2018_global_progress_report.pdf?ua=1

15. WHO Framework Convention on Tobacco Control. Report of the Eighth session of the Conference of the Parties to the WHO Framework Convention on Tobacco Control [Internet]. Eighth session Conference of the Parties to the WHO Framework Convention on Tobacco Control; 2018 Oct 1-6; Geneva, Switzerland: WHO; 2018 [cited 2020 Apr 30]. Available from: https://www.who.int/ fctc/cop/sessions/cop8/COP8_REPORT_EN.PDF

16. Ministério da Saúde (BR). Vigitel Brasil 2019: vigilância de fatores de risco e proteção para doenças crônicas por inquérito telefônico: estimativas sobre frequência e distribuição sociodemográfica de fatores de risco e proteção para doenças crônicas nas capitais dos 26 estados brasileiros e no Distrito Federal em 2019 [Internet]. Brasília, DF: Ministério da Saúde; 2020 [acesso 2020 abr 29]. Disponível em: https://portalarquivos.saude.gov.br/ images/pdf/2020/April/27/vigitel-brasil-2019-vigilanciafatores-risco.pdf

17. José BPS, Corrêa RA, Malta DC, et al. Mortalidade e incapacidade por doenças relacionadas à exposiçáo ao tabaco no Brasil, 1990 a 2015. Rev Bras Epidemiol. 2017;20(Suppl 1):75-89. doi: https://doi. org/10.1590/1980-5497201700050007

18. Levy D, de Almeida LM, Szklo A. The Brazil SimSmoke policy simulation model: the effect of strong tobacco control policies on smoking prevalence and smokingattributable deaths in a middle income nation. PLoS Med. 2012;9(11):e1001336. doi: https://doi. org/10.1371/journal.pmed.1001336

19. Cavalcante TM, Pinho MCM, Perez CA, et al. Brasil: balanço da Política Nacional de Controle do Tabaco na última década e dilemas. Cad Saúde Pública. 2017;33(Suppl 3):e00138315. doi: https://doi. org/10.1590/0102-311x00138315 
20. Bialous SA, Presman S, Gigliotti A, et al. A resposta da indústria do tabaco à criação de espaços livres de fumo no Brasil. Rev Panam Salud Publica [Internet]. 2010 [acesso 2020 abr 20];27(4):283-90. Disponível em: https://scielosp.org/pdf/rpsp/2010.v27n4/283-290/pt

21. Cancian N. Entidades se armam para batalha judicial sobre cigarro com sabor. Folha de São Paulo [Internet]. 2018 fev 02 [acesso 2020 abr 22]. Disponível em: https:// www1.folha.uol.com.br/equilibrioesaude/2018/02/ entidades-se-armam-para-batalha-judicial-sobre-cigarrocom-sabor.shtml

22. Confederação Nacional de Municípios [Internet]. Brasília, DF: CNM; c2016. Plenário mantém tramitação de projeto que amplia combate ao tabagismo; 2018 jun 21. [acesso 2020 abr 20]. Disponível em: https:// www.cnm.org.br/comunicacao/noticias/plenariomantem-tramitacao-de-projeto-que-amplia-combateao-tabagismo

23. Wakefield M, Morley C, Horan JK, et al. The cigarette pack as image: new evidence from tobacco industry documents. Tob Control. 2002;11(Suppl 1):i73-i80. doi: https://doi.org/10.1136/tc.11.suppl_1.i73

24. Kotnowski K, Hammond D. The impact of cigarette pack shape, size and opening: evidence from tobacco company documents. Addiction. 2013;108(9):1658-68. doi: https://doi.org/10.1111/add.12183

25. Doxey L, Hammond D. Deadly in pink: the impact of cigarette packaging among young women. Tob Control. 2011;20(5):353-60. doi: https://doi.org/10.1136/ tc. 2010.038315 